\title{
Theoretical and Numerical Examination of Equivalent Resistances in Zone-Control Induction Heating System
}

\author{
Teruyoshi Sasayama ${ }^{* a)}$ Member, $\quad$ Yusuke Yanamoto** Member \\ Shunsuke Funaji** $\quad$ Non-member, Takahiro Ao ${ }^{* * * *} \quad$ Non-member
}

(Manuscript received April 14, 2014, revised July 22, 2014)

\begin{abstract}
When a workpiece is heated by eddy currents using a zone-control induction heating $(\mathrm{ZCIH})$ system, there exists both inductance and resistance in the induction heating circuit. To efficiently control a ZCIH system, the detailed behavior of the self inductance and equivalent resistance of each coil and the mutual inductance and equivalent resistance between the coils should be clarified beforehand. This paper proposes the concept of the self- and mutual-equivalent resistances in the eddy current circuit and discusses the theoretical physical meaning and properties of these parameters. We also derive a theoretical formula for these parameters using a simple assumption and then examine their properties.
\end{abstract}

Keywords: coils, eddy current, electromagnetic field, finite element method, induction heating

\section{Introduction}

A zone-control induction heating $(\mathrm{ZCIH})$ system, which has several coils and where each coil is connected to an independent power supply, has been proposed previously ${ }^{(1)-(4)}$. Conventional induction heating systems may not uniformly heat $^{(5)}$, whereas a ZCIH system can uniformly heat workpiece by controlling the amplitude of the current under the condition that the frequency and phase angle of the current in each coil are the same.

When the workpiece is heated by an eddy current caused by the current in the coil, the coil can be treated as both inductance and resistance. The resistance that corresponds to the heat in the workpiece can be treated as an "equivalent resistance", which can be classified as "self-equivalent resistance" and "mutual-equivalent resistance". A self-equivalent resistance appears in a coil when a current is applied to the coil, while a mutual-equivalent resistance appears when a current is applied to the other coil. The eddy current in the workpiece can be explained by the equivalent circuit model using equivalent resistances.

To appropriately control the ZCIH system, the self- and

a) Correspondence to: Teruyoshi Sasayama. E-mail: sasayama@ kyushu-u.ac.jp

* Department of Electrical and Electronic Engineering, Kyushu University

744, Motooka, Nishi-ku, Fukuoka 819-0395, Japan

** Railroad Products Company, Nabtesco Corporation JA Kyosai Bldg., 2-7-9, Hirakawacho, Chiyoda-ku, Tokyo 102-0093, Japan

*** Machinery \& System Hq., Mitsui Engineering \& Shipbuilding Co. Ltd.

3-16-3, Tamahara, Tamano 706-0014, Japan

**** Engineering Department, MES Power-Electronics Industry Co. Ltd.

3-1-1, Tama, Tamano 706-8651, Japan mutual-equivalent resistances should be obtained. These resistances can be calculated using finite element methods (FEM). However, it is difficult to understand the characteristics of the resistances unless they are theoretically investigated.

When the size of the coils and workpiece are optimized, the computational cost should decrease to iteratively calculate the various circuit parameters such as inductance and equivalent resistance. Moreover, the resistivity of the workpiece varies as the temperature and circuit parameters change. In such a case, the circuit parameters should be calculated in real time. However, it is difficult to compute the circuit parameters and control in real time using FEM because of the high computational cost. To overcome these issues, a simplified circuit model is required to calculate the circuit parameters. One of the calculation methods of the equivalent resistances has been proposed ${ }^{(6)}$. On the other hand, the concept of equivalent resistance, especially mutual-equivalent resistance, has not been fully understood. Understanding the theory behind equivalent resistances is essential to optimize the design of the ZCIH system.

In this paper, we propose the concept of using self- and mutual-equivalent resistances for the precise control of a $\mathrm{ZCIH}$ system. We derive the theoretical formula of these parameters using a simple assumption, and then examine their properties.

\section{Self- and Mutual-equivalent Resistances}

2.1 For a Single Coil In accordance with the derivation of the classical eddy current loss ${ }^{(7)}$, we derive the eddy current loss of the brick workpiece shown in Fig. 1. The inductance in the eddy current circuit is assumed to be sufficiently smaller than that in the exciting circuit, and the magnetic reaction field is neglected. From Faraday's law of induction, the interlinkage flux induced by the eddy current 


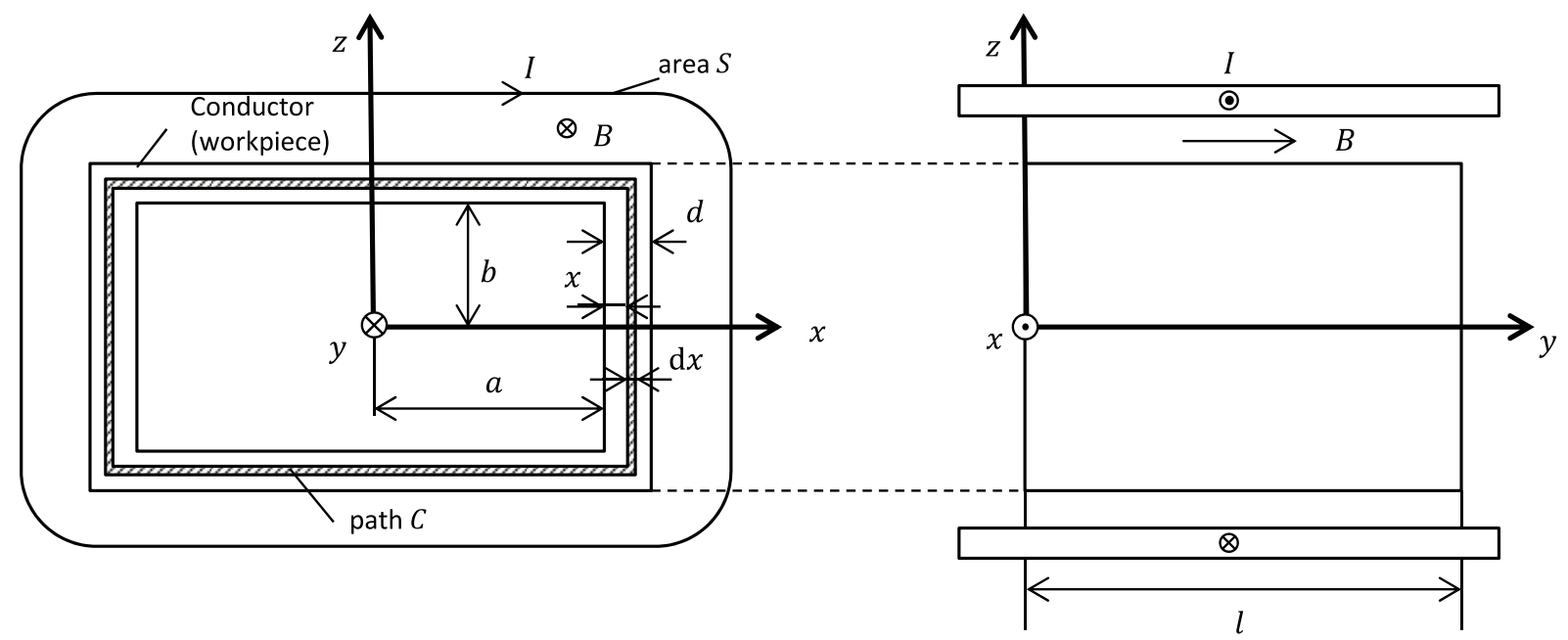

Fig. 1. Brick workpiece of which the inside is vacant

$\Phi_{\mathrm{e}}(t)$ is proportional to $\mathrm{d} \Phi(t) / \mathrm{d} t$. Therefore, $\Phi_{\mathrm{e}}(t)$ can be expressed as follows:

$$
\Phi_{\mathrm{e}}(t)=-\kappa \frac{\mathrm{d} \Phi(t)}{\mathrm{d} t}
$$

where $\kappa$ is the proportional coefficient. The interlinkage flux in the coil is obtained as follows:

$$
\Phi(t)+\Phi_{\mathrm{e}}(t)=L i(t)-\kappa L \frac{\mathrm{d} i(t)}{\mathrm{d} t}
$$

where $L$ is the inductance and $i(t)$ is the current $(\Phi(t)=L i(t))$.

Then, when the current is sinusoidal, the induced voltage is obtained as follows:

$$
\begin{aligned}
v(t) & =\frac{\mathrm{d}\left(\Phi(t)+\Phi_{\mathrm{e}}(t)\right)}{\mathrm{d} t} \\
& =\left(\mathrm{j} \omega L+\omega^{2} \kappa L\right) \cdot \sqrt{2} I e^{\mathrm{j} \omega t} \\
& =\left(\mathrm{j} \omega L+\omega^{2} \kappa L\right) \cdot i(t) \cdots
\end{aligned}
$$

where $\omega$ is the angular frequency of the current, $I$ is the root mean square value of the current in the induced coil, and $\mathrm{j}=\sqrt{-1}$. Let us define $\omega^{2} \kappa L$ in (3) as $R$, which is called as a "self-equivalent resistance". When a phasor is used in (3), the following equation is obtained:

$$
\dot{V}=(\mathrm{j} \omega L+R) \dot{I}
$$

where dot $(\cdot)$ above variables means the complex number followed by the phasor method.

We consider the eddy current loss of the specimen $W_{\mathrm{e}}^{*}$ shown in Fig. 1. Here, the sinusoidal magnetic flux is assumed to be applied through the y-axis direction.

The flux is described as follows:

$$
\begin{aligned}
\Phi(t) & =B \cdot 2(x+a) \cdot 2(x+b) \\
& =4(x+a)(x+b) B_{\max } \cos \omega t
\end{aligned}
$$

where $B=B_{\max } \cos \omega t$ is the magnetic flux density and $B_{\max }$ is the maximum flux density.

The induced voltage $e$ along pass $C$ is obtained using Faraday's law as follows:

$$
e=4(x+a)(x+b) \omega B_{\max } \sin \omega t
$$

Hence, the effective voltage is obtained as follows:

$$
E=2 \sqrt{2}(x+a)(x+b) \omega B_{\max }
$$

The length of pass $C$ is $4(x+a)+4(x+b)$ and the crosssection area is $l \mathrm{~d} x$. Therefore, the resistance of the shaded area in Fig. 1, $\mathrm{d} R$, is

$$
\mathrm{d} R=\frac{4(x+a)+4(x+b)}{\sigma l \mathrm{~d} x}
$$

where $\sigma$ is the electrical conductivity. Subsequently, the eddy current loss $d W_{\mathrm{e}}^{*}$ in the shaded area shown in Fig. 1 is obtained as follows:

$$
\begin{aligned}
\mathrm{d} W_{\mathrm{e}}^{*} & =\frac{E^{2}}{\mathrm{~d} R} \\
& =\sigma l \omega^{2} B_{\max }^{2} \frac{(x+a)^{2}(x+b)^{2}}{x+\frac{a+b}{2}} \mathrm{~d} x
\end{aligned}
$$

Integrating from 0 to $d$ about $x$ in (9), the eddy current loss of the workpiece $W_{\mathrm{e}}^{*}$ is obtained as follows:

$$
\begin{aligned}
W_{\mathrm{e}}^{*}= & \sigma l \omega^{2} B_{\max }^{2}\left[\frac{(a+b+2 d)^{4}-(a+b)^{4}}{64}\right. \\
& -\frac{(a-b)^{2}}{16}\left\{(a+b+2 d)^{2}-(a+b)^{2}\right\} \\
& \left.+\frac{(a-b)^{4}}{16} \log \frac{a+b+2 d}{a+b}\right] \ldots \ldots \ldots
\end{aligned}
$$

In general, the power loss includes not only the eddy current loss but also the copper loss. The eddy current loss and the copper loss are independent. Therefore, the copper loss is omitted in this paper to focus only on the equivalent resistance.

Considering the relation between $B_{\max }$ and $I$, the following relationship is obtained:

$$
\begin{aligned}
B_{\max } & =\frac{\Phi_{\max }}{S N} \\
& =\frac{L I_{\max }}{S N} \\
& =\frac{\sqrt{2} L I}{S N}
\end{aligned}
$$




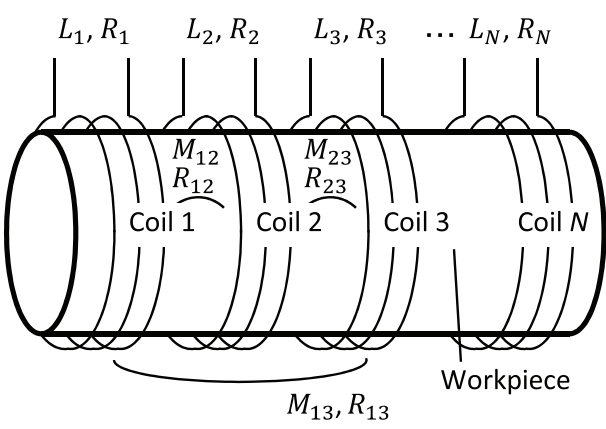

(a) Arrangement of coils and a workpiece

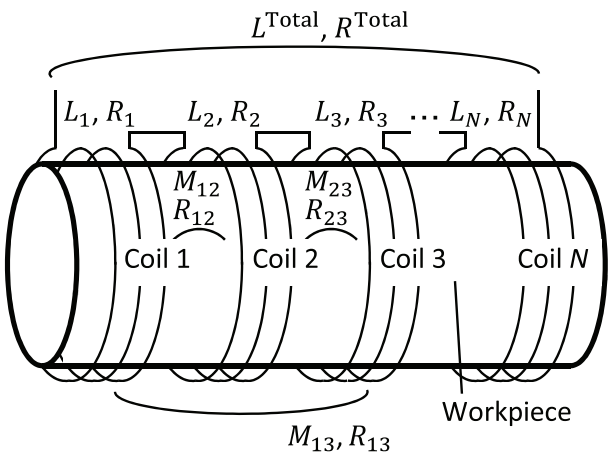

(b) Connection in series

Fig. 2. Multiple Coils

where $\Phi_{\max }$ is the maximum value of flux, $S$ is the area of the coil, $N$ is the number of turns in the coil, and $I_{\max }$ is the maximum current of the current in the induced coil, respectively.

Considering $W_{\mathrm{e}}^{*}$ equals the power loss at the equivalent resistance, the following relationship is obtained:

$$
W_{\mathrm{e}}^{*}=R I^{2}
$$

Then the equivalent resistance $R$ is finally obtained as follows:

$$
\begin{aligned}
R= & \frac{2 \sigma l \omega^{2} L^{2}}{S^{2} N^{2}}\left[\frac{(a+b+2 d)^{4}-(a+b)^{4}}{64}\right. \\
& -\frac{(a-b)^{2}}{16}\left\{(a+b+2 d)^{2}-(a+b)^{2}\right\} \\
& \left.+\frac{(a-b)^{4}}{16} \log \frac{a+b+2 d}{a+b}\right] \ldots \ldots \ldots
\end{aligned}
$$

2.2 For Multiple Coils When there are a lot of coils as shown in Fig. 2(a), following the same manner, the following equation is obtained:

$$
\begin{aligned}
\Phi= & \Phi_{m}+\Phi_{\mathrm{e} m}+\sum_{n ; m \neq n}\left(\Phi_{m n}+\Phi_{\mathrm{e} m n}\right) \\
= & L_{m} i_{m}-\kappa_{m} L_{m} \frac{\mathrm{d} i_{m}}{\mathrm{~d} t} \\
& +\sum_{n ; m \neq n}\left(M_{m n} i_{n}-\kappa_{m n} M_{m n} \frac{\mathrm{d} i_{n}}{\mathrm{~d} t}\right)
\end{aligned}
$$

where $L_{m}$ is the inductance of the coil $m, M_{m n}$ is the mutual inductance, $i_{m}$ is the current in coil $m, \Phi_{m}$ is the interlinkage flux in coil $m$ induced by itself, $\Phi_{m n}$ is the interlinkage flux in coil $m$ induced by coil $n, \Phi_{\mathrm{e} m}$ is the interlinkage flux in coil $m$ induced by the eddy current, $\Phi_{\mathrm{e} m n}$ is the interlinkage flux in coil $m$ induced by the eddy current that is generated by coil $n$,

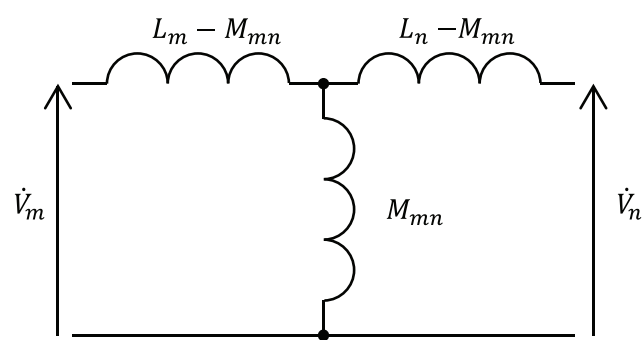

(a) Without eddy current

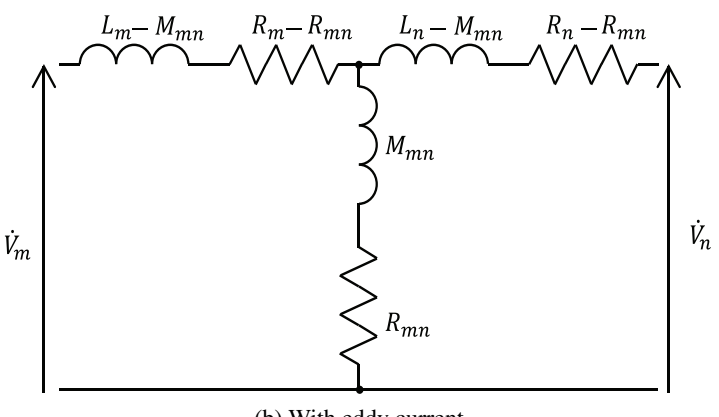

(b) With eddy current

Fig. 3. Equivalent circuit of a transformer

and $\kappa_{m}$ and $\kappa_{m n}$ are the proportional coefficients corresponding to coil $m$ and coils $m$ and $n$, respectively, and the explicit notation of $(t)$ is omitted.

When $\omega^{2} \kappa_{m} M_{m}$ and $\omega^{2} \kappa_{m n} M_{m n}$ are defined as $R_{m}$ that is called the "self-equivalent resistance" and $R_{m n}$ that is called the "mutual-equivalent resistance", respectively, the following equation is obtained:

$$
\dot{V}_{m}=\left(\mathrm{j} \omega L_{m}+R_{m}\right) I_{m}+\sum_{n ; m \neq n}\left(\mathrm{j} \omega M_{m n}+R_{m n}\right) \dot{I}_{n}
$$

If $\mathrm{j} \omega L_{m}$ and $\mathrm{j} \omega M_{m n}$ are substituted for $\left(\mathrm{j} \omega L_{m}+R_{m}\right)$ and $\left(\mathrm{j} \omega M_{m n}+R_{m n}\right)$ in (15), respectively, the equation corresponds to the well-known equation of a transformer. If all the coils are connected in series as shown in Fig. 2(b), considering reciprocity, the total inductance $L^{\text {Total }}$ and the total equivalent resistance $R^{\text {Total }}$ are obtained as follows:

$$
\begin{aligned}
L^{\text {Total }} & =\sum_{m} L_{m}+\sum_{m, n ; m \neq n} M_{m n} \\
& =\sum_{m} L_{m}+2 \sum_{m, n ; m<n} M_{m n} \\
R^{\text {Total }} & =\sum_{m} R_{m}+\sum_{m, n ; m \neq n} R_{m n} \\
& =\sum_{m} R_{m}+2 \sum_{m, n ; m<n} R_{m n}
\end{aligned}
$$

\section{Equivalent circuit model of ZCIH}

The equivalent circuit of a transformer is shown in Fig. 2(a). In accordance with the result obtained in Section II, substituting $\mathrm{j} \omega L_{m}, \mathrm{j} \omega L_{n}$, and $\mathrm{j} \omega M_{m n}$ as $\left(\mathrm{j} \omega L_{m}+R_{m}\right)$, $\left(\mathrm{j} \omega L_{n}+R_{n}\right)$, and (j $\left.\omega M_{m n}+R_{m n}\right)$ in Fig. 3(a), respectively, the equivalent circuit of a transformer with eddy currents is obtained as shown in Fig. 3(b).

The equivalent circuit of a transformer in Fig. 3(b) has been 
reported ${ }^{(6)(8)}$. However, the reason for connecting the inductance and resistance in series has not been reported. In contrast, the theory described in Section II explains the reason for them to be connected in series.

\section{Inductances and Equivalent Resistances of ZCIH}

4.1 Definition of Coupling Coefficient The inductance coupling coefficient between coils $m$ and $n, k_{m n}$ is expressed as follows:

$$
k_{m n}=\frac{M_{m n}}{\sqrt{L_{m} L_{n}}} .
$$

Similarly, to consider the relationship between the selfequivalent resistances and the mutual-equivalent resistance, in this paper, we introduce the equivalent resistance coupling coefficient between coils $m$ and $n, k_{m n}^{\mathrm{R}}$ defined as follows:

$$
k_{m n}^{\mathrm{R}}=\frac{R_{m n}}{\sqrt{R_{m} R_{n}}}
$$

When the current $I$ flows in coil $m$, the eddy current loss is proportional to the square of the flux density, under the assumption that the area of the flux is constant. Therefore, the eddy current loss $W_{m}$ is expressed as follows:

$$
W_{m}=R_{m} I^{2}=\xi_{m} \phi_{m}^{2}
$$

where $\xi_{m}$ is the proportional constant, $\phi_{m}$ is the flux of the interlinkage flux in coil $m$ when the current $I$ flows. Substituting (17) and (20) into (19), the equivalent resistance coupling coefficient $k_{m n}^{\mathrm{R}}$ is obtained as follows:

$$
k_{m n}^{\mathrm{R}}=\frac{R_{m n} I^{2}}{\sqrt{R_{m} I^{2} \cdot R_{n} I^{2}}}=\frac{W_{m, n}^{\text {Total }}-W_{m}-W_{n}}{2 \sqrt{W_{m} W_{n}}} \cdots \cdots
$$

where $W_{m, n}^{\text {Total }}$ is the eddy current loss when the current $I$ passes through coils $m$ and $n$.

4.2 Example of Calculation of Coupling Coefficient of Equivalent Resistances In this study, we assume three coils and three workpieces as shown in Fig. 4. The inductance coupling coefficient between coils $m$ and $n$ is defined as $k_{m n}$, and the inductance coupling coefficient between workpiece $m$ and coil $n$ is defined as $k_{\bar{m} n}$.

When the current $I$ flows thorough coil 1, considering that the fluxes passing in coil 1,2 , and 3 are $k_{\overline{1} 1} \phi_{1}, k_{\overline{2} 1} \phi_{1}$, and $k_{\overline{3} 1} \phi_{1}$, as shown in Fig. 5(a), respectively, the eddy current loss $W_{1}$ is described as follows:

$$
W_{1}=\xi_{1}\left(k_{\overline{1} 1} \phi_{1}\right)^{2}+\xi_{2}\left(k_{\overline{2} 1} \phi_{1}\right)^{2}+\xi_{3}\left(k_{\overline{3} 1} \phi_{1}\right)^{2} \cdots \cdots
$$

Similarly, when the current $I$ flows thorough coil 2 and 3 as shown in Fig. 5(b), the eddy current loss $W_{2}$ and $W_{3}$ are described as follows:

$$
\begin{aligned}
& W_{2}=\xi_{1}\left(k_{\overline{1} 2} \phi_{2}\right)^{2}+\xi_{2}\left(k_{\overline{2} 2} \phi_{2}\right)^{2}+\xi_{3}\left(k_{\overline{3} 2} \phi_{2}\right)^{2} \cdots \\
& W_{3}=\xi_{1}\left(k_{\overline{1} 3} \phi_{3}\right)^{2}+\xi_{2}\left(k_{\overline{2} 3} \phi_{3}\right)^{2}+\xi_{3}\left(k_{\overline{3} 3} \phi_{3}\right)^{2} \cdots
\end{aligned}
$$

When the current $I$ flows through coils 1 and 2 , the interlinkage fluxes in coils 1,2 , and 3 are $k_{\overline{1} 1} \phi_{1}+k_{\overline{1} 2} \phi_{2}$, $k_{\overline{2} 1} \phi_{1}+k_{\overline{2} 2} \phi_{2}$, and $k_{\overline{3} 1} \phi_{1}+k_{\overline{3} 2} \phi_{2}$ as shown in Fig. 4(c), respectively, and the total eddy current loss $W_{1,2}^{\text {Total }}$ is derived as follows:

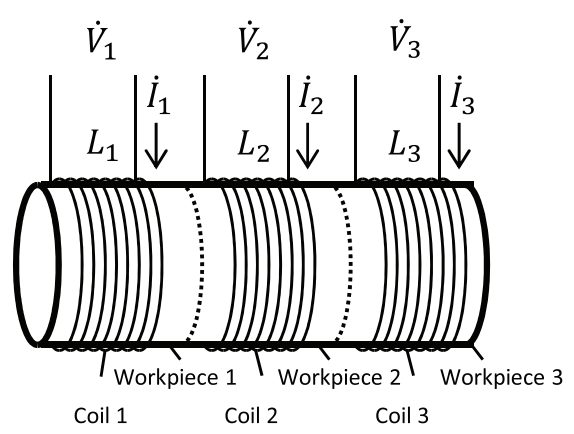

Fig. 4. Three coils and workpieces

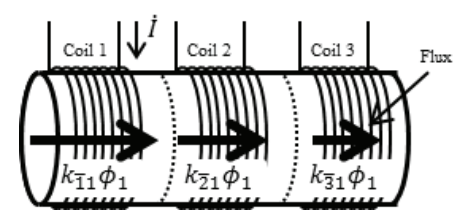

(a) Applying current to coil 1 only
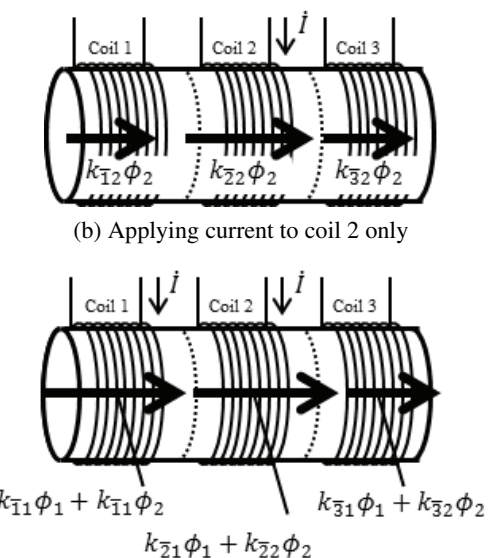

(c) Applying current to coil 1 and 2

Fig. 5. Flux distribution in coils

$$
\begin{aligned}
W_{1,2}^{\text {Total }}= & \xi_{1}\left(k_{\overline{1} 1} \phi_{1}+k_{\overline{1} 2} \phi_{2}\right)^{2} \\
& +\xi_{2}\left(k_{\overline{2} 1} \phi_{1}+k_{\overline{2} 2} \phi_{2}\right)^{2} \\
& +\xi_{3}\left(k_{\overline{3} 1} \phi_{1}+k_{\overline{3} 2} \phi_{2}\right)^{2} .
\end{aligned}
$$

When the current $I$ flows through coils 1 and 3 , the interlinkage fluxes in coils 1,2 , and 3 are $k_{\overline{1} 1} \phi_{1}+k_{\overline{1} 3} \phi_{3}$, $k_{\overline{2} 1} \phi_{1}+k_{\overline{2} 3} \phi_{3}$, and $k_{\overline{3} 1} \phi_{1}+k_{\overline{3} 3} \phi_{3}$, respectively, and the total eddy current loss $W_{1,3}^{\text {Total }}$ is derived as follows:

$$
\begin{aligned}
W_{1,3}^{\text {Total }}= & \xi_{1}\left(k_{\overline{1} 1} \phi_{1}+k_{\overline{1} 3} \phi_{3}\right)^{2} \\
& +\xi_{2}\left(k_{\overline{2} 1} \phi_{1}+k_{\overline{2} 3} \phi_{3}\right)^{2} \\
& +\xi_{3}\left(k_{\overline{3} 1} \phi_{1}+k_{\overline{3} 3} \phi_{3}\right)^{2} .
\end{aligned}
$$

If the workpiece is sufficiently thin and close to the coil, and the relative magnetic permeability of the workpiece approaches unity, the following relationships are obtained:

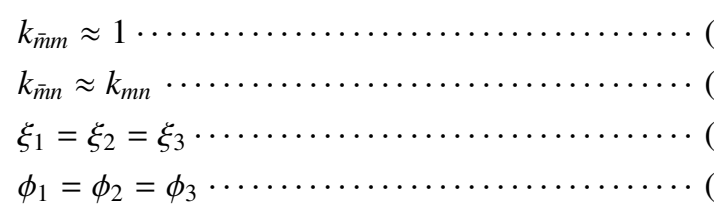

Moreover, substituting (22)-(30) into (21) and considering $k_{12}=k_{13}$, the following equations are obtained: 


$$
\begin{aligned}
& k_{12}^{\mathrm{R}}=\frac{2 k_{12}+k_{12} k_{13}}{\sqrt{\left(k_{12}^{2}+k_{13}^{2}+1\right)\left(2 k_{12}^{2}+1\right)}} . \\
& k_{13}^{\mathrm{R}}=\frac{k_{12}^{2}+2 k_{13}}{k_{12}^{2}+k_{13}^{2}+1} \cdots \ldots \ldots \ldots .
\end{aligned}
$$

Note that the equivalent resistance coupling coefficient is not equal to the inductance coupling coefficient. On the other hand, the former can be derived using the latter.

4.3 Calculation of Inductances and Resistances using FEM The method to calculate the inductances and resistances is briefly described in this subsection ${ }^{(9)}$.

The flux linkage $\dot{\Phi}$ of the coil is given by

$$
\dot{\Phi}=\frac{n}{S} \iiint_{V} \boldsymbol{A} \cdot \boldsymbol{n}_{s} \mathrm{~d} V
$$

where $S$ is the cross-sectional area of coil, $n$ is the number of turns of coil, $\boldsymbol{A}$ is the magnetic vector potential, and $\boldsymbol{n}_{s}$ is the unit vector parallel to the current.

The induced voltage $\dot{V}$ of the coil is given by

$$
\dot{V}=-\mathrm{j} \omega \dot{\Phi}
$$

By using the induced voltage $\dot{V}_{1}$ in the coil 1 and the current $\dot{I}_{1}$ fed in the coil 1 , the following equation is obtained:

$$
\begin{aligned}
\dot{Z}_{m} & =\frac{\dot{V}_{m}}{\dot{I}_{m}} \\
& =R_{m}+\mathrm{j} \omega L_{m}
\end{aligned}
$$

where

$$
R_{m}=\operatorname{Re}\left[\frac{\dot{V}_{m}}{\dot{I}_{m}}\right], \quad L_{m}=\frac{1}{\omega} \cdot \operatorname{Im}\left[\frac{\dot{V}_{m}}{\dot{I}_{m}}\right] \ldots
$$

and $\operatorname{Re}[\cdot]$ and $\operatorname{Im}[\cdot]$ denote the real part and imaginary part in the square brackets.

By using the induced voltage $\dot{V}_{m}$ in a coil $m$ and the current $\dot{I}_{n}$ fed in the coil $n$, the following equations are obtained:

$$
\begin{aligned}
\dot{Z}_{m n} & =\frac{\dot{V}_{m}}{\dot{I}_{n}} \\
& =R_{m n}+\mathrm{j} \omega M_{m}
\end{aligned}
$$

where

$$
R_{m n}=\operatorname{Re}\left[\frac{\dot{V}_{m}}{\dot{I}_{n}}\right], \quad M_{m n}=\frac{1}{\omega} \cdot \operatorname{Im}\left[\frac{\dot{V}_{m}}{\dot{I}_{n}}\right] \ldots \ldots \ldots
$$

Our previous study ${ }^{(10)}$ shows that the self- and mutualequivalent resistance and inductance calculated by FEM are almost identical to the experimental results.

\section{Results}

5.1 Self-Equivalent Resistance Figure 6 shows the analyzed ZCIH model. Each coil has six turns. The workpiece is assumed to be graphite, which has a relative permeability of unity. The resistivity $\rho$ at room temperature and at $700^{\circ} \mathrm{C}$ are $1200 \mu \Omega \cdot \mathrm{cm}$ (electrical conductivity: $\sigma=$ $8.3 \times 10^{4} \mathrm{~S} / \mathrm{m}$ ) and $900 \mu \Omega \cdot \mathrm{cm}$ (electrical conductivity: $\sigma=$ $\left.1.1 \times 10^{5} \mathrm{~S} / \mathrm{m}\right)$, respectively. In FEM analysis, the eddy current of the coil was neglected.

Table 1 lists $R_{1}$ and $R_{2}$ calculated using FEM and estimated using (10) when the resistivity is $1200 \mu \Omega \cdot \mathrm{cm}$ and the frequency of the current is $35 \mathrm{kHz}$. The estimated $R_{1}$ and $R_{2}$ are almost the same as those calculated by FEM.

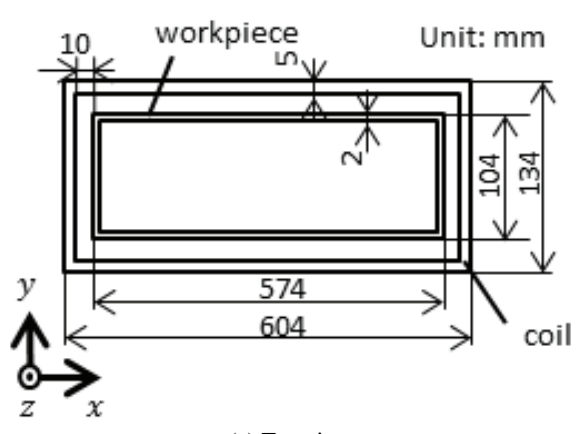

(a) Top view

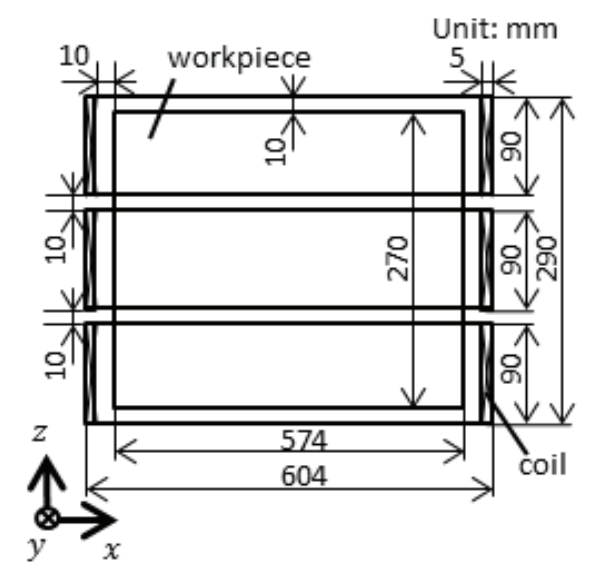

(b) Front view

Fig. 6. Analyzed model of ZCIH system

Table 1. Self-equivalent resistance of coil 1

\begin{tabular}{c|c|c}
\hline & $\begin{array}{c}\text { Room } \\
\text { temperature }\end{array}$ & $700^{\circ} \mathrm{C}$ \\
\hline FEM & 1.08 & 1.03 \\
\hline Estimate & 1.13 & 1.19 \\
\hline
\end{tabular}

5.2 Coupling Coefficients The frequency of the current was set to $1 \mathrm{~Hz}-1 \mathrm{MHz}$ and resistivity of workpiece was chosen as $1200 \mu \Omega \cdot \mathrm{cm}$. $k_{m n}^{\mathrm{R}}$ was calculated using FEM, and estimated using (31) and (32). In (31) and (32), $k_{m n}$ was calculated by FEM.

Figure 7 shows the frequency characteristics of $k_{m n}, k_{m n}^{\mathrm{R}}$ (FEM), and $k_{m n}^{\mathrm{R}}$ (Estimated). $k_{m n}^{\mathrm{R}}$ (FEM) and $k_{m n}^{\mathrm{R}}$ (Estimated) changes in the range $1 \mathrm{~Hz}-1 \mathrm{MHz}$. A fairly good agreement between $k_{m n}^{\mathrm{R}}(\mathrm{FEM})$ and $k_{m n}^{\mathrm{R}}$ (Estimated) is obtained. This result indicates that the relationships between $k_{m n}$ and $k_{m n}^{\mathrm{R}}$ as shown in (31) and (32) are satisfied although $k_{m n}$ varies owing to the change in frequency.

\section{Discussion}

In Fig. 7, the equivalent-coupling coefficients estimated by using (31) and (32) are approximately $40 \%$ smaller than those calculated using FEM when the frequency of the current was $10 \mathrm{kHz}-100 \mathrm{kHz}$. The difference is considered to be caused by the approximation that the self inductance of the coil equals that of the workpiece under the coil, and the mutual inductance between the coil and workpiece equals that between the coils in (27) and (28). If a method that does not require such approximations is developed, the accuracy of the equivalent-resistance coupling coefficients would be enhanced. 


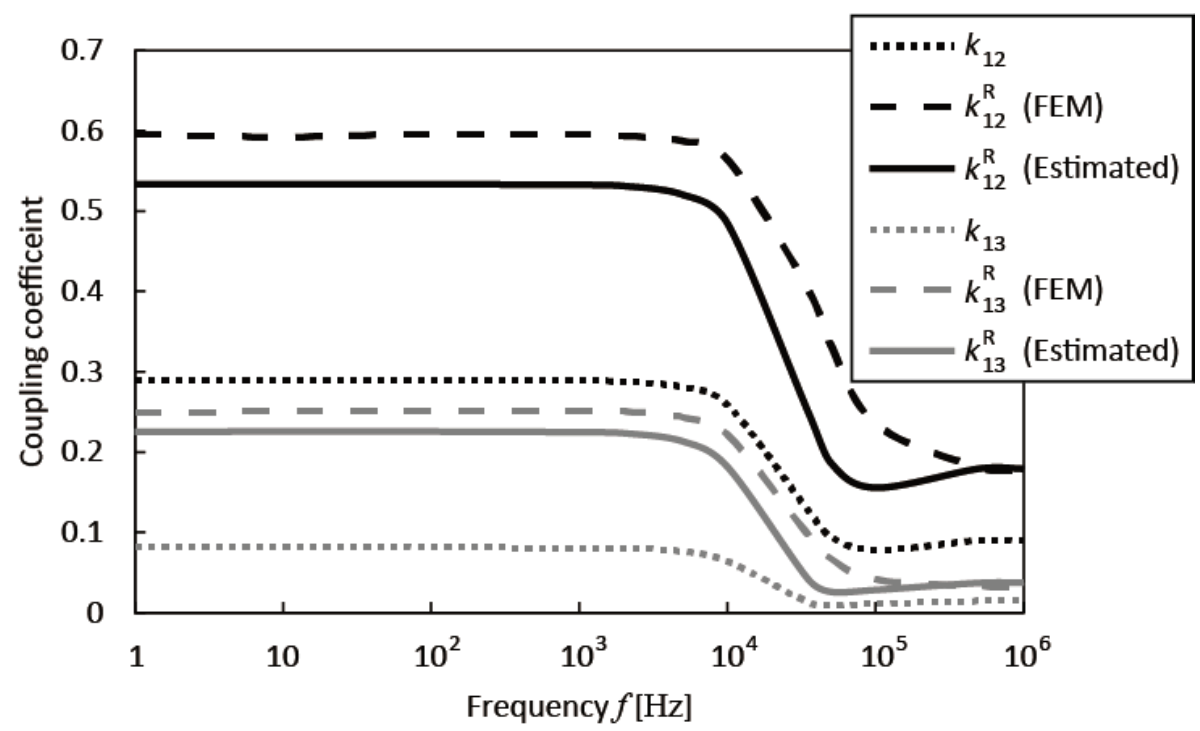

Fig. 7. Frequency characteristics of inductance coupling coefficient and equivalent resistance coupling coefficient

In this study, the equivalent-resistance coupling coefficients were obtained using the result of the inductance coupling coefficients calculated by FEM. On the other hand, the method used to calculate self and mutual inductance has been reported previously ${ }^{(11)-(13)}$. In the future work, we will derive the self and mutual inductance of a ZCIH system, using these methods to reduce the computational cost required to obtain resistance coupling coefficients, i.e., without using FEM. Furthermore, we will improve this model to enable consideration of the effect of the magnetic reaction field.

\section{Conclusions}

We propose the concept of self- and mutual-equivalent resistance in eddy current circuits, and discuss the theoretical physical meaning and properties of these parameters. We also derive the theoretical formula for these parameters using a simple assumption and then examine their properties. Our findings will contribute to optimize design and control of the ZCIH system and reduce of the computation cost of calculating the electromagnetic parameters.

\section{Acknowledgment}

The authors would like to thank the late Prof. Norio Takahashi of Okayama University for his excellent instruction and very kind support. The authors wish to thank Mr. Naoki Uchida, a retired researcher of Mitsui Engineering \& Shipbuilding Co. Ltd. for his useful advice.

\section{References}

(1) D. Miyagi, A. Saitou, N. Takahashi, N. Uchida, and K. Ozaki: "Improvement of Zone Control Induction Heating Equipment for High-speed Processing of Semiconductor Devices", IEEE Trans. Magn., Vol.42, No.2, pp.292-294 (2006)

( 2 ) H. Kurose, D. Miyagi, N. Takahashi, N. Uchida, and K. Kawanaka: "3-D Eddy Current Analysis of Induction Heating Apparatus Considering Heat Emission, Heat Conduction, and Temperature Dependence of Magnetic Characteristics", IEEE Trans. Magn., Vol.45, No.3, pp.1847-1850 (2009)

( 3 ) H. Kagimoto, D. Miyagi, N. Takahashi, N., Uchida, and K. Kawanaka: "Effect of Temperature Dependence of Magnetic Properties on Heating Characteristics of Induction Heater", IEEE Trans. Magn., Vol.46, No.8, pp.30183021 (2010)
( 4 ) H.N. Pham, H. Fujita, K. Ozaki, and N. Uchida: "Dynamic Analysis and Control for Resonant Currents in a Zone-Control Induction Heating System", IEEE Trans. Power Electron., Vol.28, No.3, pp.1297-1307 (2013)

( 5 ) W. Hurley and J.G. Kassakian: "Induction heating of circular ferromagnetic plates", IEEE Trans. Magn., Vol.15, No.4, pp.1174-1181 (1979)

( 6 ) H.N. Pham, H. Fujita, K. Ozaki, and N. Uchida: "Estimating Method of Heat Distribution Using 3-D Resistance Matrix for Zone-Control Induction Heating Systems", IEEE Trans. Power Electron., Vol.27, No.7, pp.3374-3382 (2012)

( 7 ) J.P. Bastos and N. Sadowski: "Electromagnetic Modeling by Finite Element Methods", Marrcel Dekker Inc. (2003)

( 8 ) H.N. Pham, H. Fujita, K. Ozaki, and N. Uchida: "Phase Angle Control of High-Frequency Resonant Currents in a Multiple Inverter System for ZoneControl Induction Heating", IEEE Trans. Power Electron., Vol.26, No.11, pp.3357-3366 (2011)

( 9 ) Y. Yanamoto, T. Sasayama, N. Takahashi, N. Uchida, T. Ao, K. Kawanaka, and N. Matsunaka: "Examination on Equivalent Resistance and Coupling Coefficient of Zone-Control Induction Heating by Finite Element Method", Proc. Compumag 2013, PA6-16 (2013)

(10) Y. Yanamoto, T. Sasayama, S. Funaji, and T. Ao: "A study on Evaluation of Zone-control Induction Heating System by a Finite Element Method", The Papers of Technical Meeting on Static Apparatus and Rotating Machinery, IEE Japan, SA-14-23/RM-14-23, pp.37-42 (2014) (in Japanese)

(11) V. Pankrac: "The Algorithm for Calculation of the Self and Mutual Inductance of Thin-Walled Air Coils of General Shape with Parallel Axes", IEEE Trans. Magn., Vol.48, No.5, pp.1875-1889 (2012)

(12) V. Pankrac: "Generalization of Relations for Calculating the Mutual Inductance of Coaxial Coils in Terms of Their Applicability to Non-Coaxial Coils", IEEE Trans. Magn., Vol.47, No.11, pp.4552-4563 (2011)

(13) J.T. Conway: "Exact Solutions for the Mutual Inductance of Circular Coils and Elliptic Coils", IEEE Trans. Magn., Vol.48, No.1, pp.81-94 (2012) 
Teruyoshi Sasayama (Member) was born in 1983. He received his

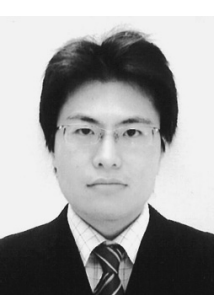
B.E. degree in electrical and electronic engineering in 2007 and his M.E. and Ph.D. degrees in electrical engineering in 2009 and 2012, respectively, from Kyoto University, Japan. He was an Assistant Professor in the Department of Electrical Engineering, Okayama University, Japan, from 2012 to 2014. Currently, he is an Assistant Professor in the Department of Electrical and Electronic Engineering, Kyushu University, Japan. He was a Research Fellow of the Japan Society for the Promotion of Science (JSPS) from April 2009 to March 2012. His research interests include magnetic field analysis using the finite element method and signal processing of electroencephalogram. Dr. Sasayama is a member of the Institute of Electrical and Electronics Engineers (IEEE), Institute of Electrical Engineers of Japan (IEEJ), and Institute of Electronics, Information and Communication Engineers (IEICE).

Yusuke Yanamoto (Member) was born in 1989. He received his

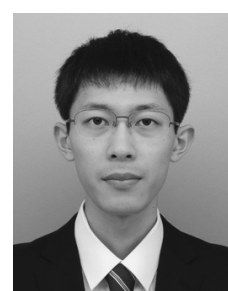

M.E. degree in Electronic and Information Systems Engineering from Okayama University in 2014

Mr. Yanamoto is a member of Institute of Electrical Engineers of Japan (IEEJ).
Shunsuke Funaji (Non-member) received the master's degree from

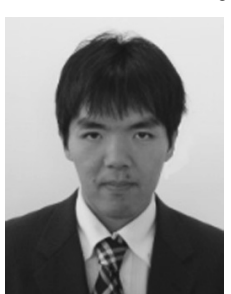
the Kanazawa University, Kanazawa, Japan in 2005. He joined the Mitsui Engineering \& Shipbuilding Co. Ltd., Tamano, Okayama, in 2005. He was involved in the development of various induction heating systems, mainly for automobile and steel industries, for 8 years.

Takahiro Ao (Non-member) received the master's degree from the

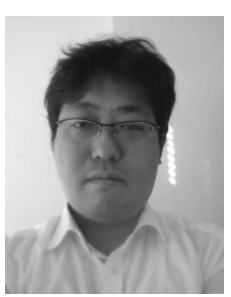
Tokushima University, Tokushima, Japan, in 1997. He joined the Mitsui Engineering \& Shipbuilding Co. Ltd., Tamano, Okayama, in 1997. He was involved in the development of various induction heating systems, mainly for automobile and steel industries, for 16 years. 